\title{
Laparoscopic Cholecystectomy: Challenges and Outcomes of the Procedure in Elderly Patients
}

\author{
Sarbjeet Singh ${ }^{1}$, Amandeep Singh $^{2}$, Ashwani Kumar ${ }^{3}$, Rohit Kapoor ${ }^{4}$, Sumit Mahajan ${ }^{5}$
}

\begin{abstract}
Introduction: Gallstone disease is more prevalent in the elderly population besides having comorbid conditions and poor physiological reserves as compared with their younger counterpart. Laparoscopic procedures have certain inherent technical limitations and unique physiological demands. Hence, the procedures may have higher complications in elderly patients and need evaluation in this age-group of patients.

Aim: This study is aimed to evaluate the safety of the procedure of laparoscopic cholecystectomy in elderly patients in terms of preoperative and intraoperative difficulties, postoperative complications, morbidity, and mortality.

Materials and methods: The study was conducted retrospectively, collecting data from hospital record of a total of 390 patients (45 elderly patients) operated during a period from 2012 to 2017.

Results: The mean age of elderly patients was 66.7 years. All the patients who underwent laparoscopic cholecystectomy had an American Society of Anesthesiologist (ASA) score of I or II. Intraoperative difficulties were encountered in $35.55 \%$ patients. Average hospital stay was 2.5 days. One patient had a minor bile leak. No mortality occurred.

Conclusion: Procedure of laparoscopic cholecystectomy is safe in elderly patients.

Keywords: Elderly patients, Laparoscopic cholecystectomy, Procedural challenges.

World Journal of Laparoscopic Surgery (2019): 10.5005/jp-journals-10033-1383
\end{abstract}

\section{INTRODUCTION}

Prevalence of gallstone disease increases with age., ${ }^{1,2}$ Further, symptoms and complications of gallstones increase with age, leading to more frequent cholecystectomies. ${ }^{3}$ As laparoscopic procedures have inherent technical limitations compared to open surgical procedures, ${ }^{4}$ the procedure of laparoscopic cholecystectomy may have higher rate of complications in elderly patients due to the frequent comorbid conditions and poor functional reserves. ${ }^{5}$ Hence, elderly patients may be considered as a separate group of patients for laparoscopic cholecystectomy. The present study is aimed to evaluate the procedure for its outcome and challenges in elderly patients.

\section{A IM}

The aim of the study is to evaluate the safety of laparoscopic cholecystectomy in elderly patients in terms of preoperative and intraoperative difficulties, postoperative complications, morbidity, and mortality.

\section{Materials and Methods}

This study was conducted retrospectively, in the Department of General Surgery, at GGS Medical College, Faridkot, Punjab. We searched the hospital record of 390 patients who underwent laparoscopic cholecystectomy during a period from 2012 to 2017. Of these 390 patients, 45 patients were found to be in elderly agegroup, i.e., $>60$ years (according to the World Health Organization definition for developing countries). Preoperative, intraoperative, and postoperative records of these patients were analyzed in terms of the ASA score, comorbid conditions, intraoperative bleeding, adhesions, difficulties of dissection, injury to organs and bile duct, operative time, conversion rate, hospital stay, and postoperative complications. The results were compared with \begin{tabular}{l}
\hline \hline${ }^{1-5}$ Department of Surgery, Guru Gobind Singh Medical College and \\
Hospital, Faridkot, Punjab, India \\
Corresponding Author: Amandeep Singh, Department of Surgery, \\
Guru Gobind Singh Medical College and Hospital, Faridkot, Punjab, \\
India, Phone: +919876201644, e-mail: singhdramandeep80@gmail. \\
com \\
How to cite this article: Singh S, Singh A, Kumar A, et al. Laparoscopic \\
Cholecystectomy: Challenges and Outcomes of the Procedure in \\
Elderly Patients. World J Lap Surg 2019;12(3):93-95. \\
Source of support: Nil \\
Conflict of interest: None
\end{tabular}

other studies. All these patients were operated under general anesthesia and surgeries were performed by trained surgeons. Procedures were performed by four port techniques, using $10 \mathrm{~mm}$, $30^{\circ}$ telescope, and using titanium clips to ligate cystic duct and cystic artery. Gallbladder was dissected from fossa using monopolar electrocautery and extracted through $10 \mathrm{~mm}$ epigastric port. Postoperatively all the patients were managed in general wards and broad-spectrum antibiotics were used for 5-7 days.

\section{Results and Discussion}

Frequency of gallstones increases after the age of 40 to become 4-10 times more in older individuals and laparoscopy is gradually replacing common surgical procedures. ${ }^{6,7}$ Our study comprises 45 elderly patients among a total of 390 patients who underwent laparoscopic cholecystectomy, during the mentioned period. In this study, the mean age of elderly patients was 66.7 years (range 61-76 years), and majority of the patients was female, with a male to female ratio of 1:2.75. When stratified for age, we observed that $82.22 \%$ patients was in $60-70$ years age-group, whereas $17.77 \%$ 
was above 70 years. Although in epidemiological studies, ${ }^{6}$ the prevalence of disease is reported to rise in conjunction with age, in our study, an inverse trend in age distribution with respect to the number of procedures (Table 1) reflects that lesser number of laparoscopic surgeries was conducted in higher age-groups. This can be justified by reports that laparoscopic surgery in elderly patients becomes more challenging due to the unique physiological demands of the procedure ${ }^{8}$ and also the ASA score (American Society of Anesthesiologists score) increases with age, increasing the risk of anesthesia. ${ }^{9,10}$ All the patients who underwent laparoscopic cholecystectomy had an ASA score of I or II, including those 17 patients $(37.77 \%)$ who had comorbid conditions, i.e., hypertension and diabetes mellitus. So this suggests the patient selection pattern, i.e., only those patients who qualified the risk criteria were selected for the procedure.

In a considerable number of patients, i.e., 16 (35.55\%), one or multiple difficulties were encountered intraoperatively as shown in the Venn diagram (Fig. 1). This has been reported in literature also that elderly patients suffer from repeated inflammation, resulting in adhering to the surrounding structures, rendering laparoscopic surgery difficult. ${ }^{11,12}$ We found that 13 patients $(28.88 \%)$ had difficult anatomy of Calot's triangle and in 5 patients (11.11\%) dense adhesions were found with omentum, colon, and duodenum. Intraoperatively uncontrolled bleeding occurred in 5 patients (11.11\%). This occurred from gallbladder fossa in two patients and cystic artery in three patients. This figure is in agreement with other series, in which the

Table 1: Distribution of patients according to age

\begin{tabular}{ll}
\hline Age-group (years) & No. of procedures \\
\hline $60-65$ & 18 \\
$66-70$ & 19 \\
$71-75$ & 6 \\
$75-80$ & 2 \\
& Total $=45$ \\
\hline
\end{tabular}

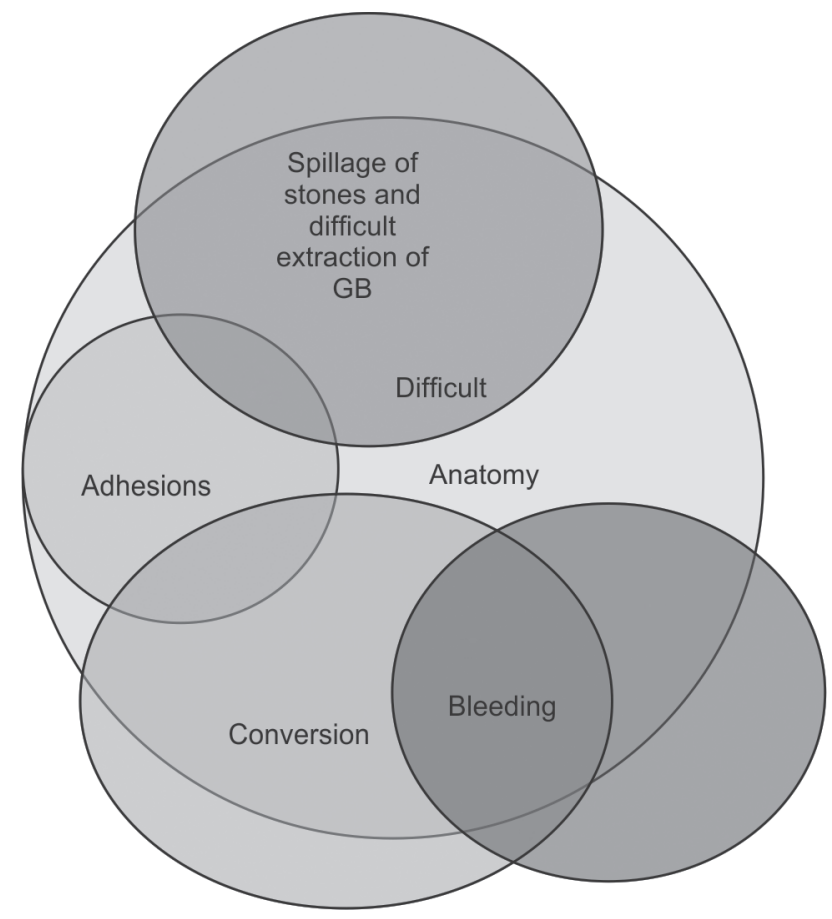

Fig. 1: Intraoperative difficulties incidence of bleed has been reported to be nearly $10 \%$, irrespective of the age of the patients. ${ }^{13}$ So this suggests that the procedure does not carry any extra risk of bleeding in elderly people. Difficulty in extraction of gallbladder was experienced in seven (15.55\%) patients. This was due to the either large number or large size of stones. Spillage of stones occurred in these patients. The procedure had to be converted to open in $13.33 \%$ ( 6 patients) and the final outcome was without any complication. This figure is higher than a standard conversion rate of $5-10 \%$, mentioned in literature ${ }^{14}$ but comparable to a figure, i.e., $14.7 \%$ mentioned in another study upon elderly patients. ${ }^{15}$ The conversion was due to multiple and concomitant intraoperative difficulties, i.e., poor visualization of anatomy, adhesions, intraoperative bleeding from cystic artery or gallbladder fossa, which has been mentioned as an important cause by other studies also. ${ }^{15}$ Operative procedure was uneventful in 29 patients. The mean operative time for all these patients was 80 minutes, which is in accordance with the mean 75 minutes in a similar study. ${ }^{16}$ This suggests that the procedure involves operative difficulties in a significant proportion of elderly patients due to the chronicity of disease. Despite operative difficulties encountered in 16 cases, in $62.5 \%$ cases operative challenges could be successfully managed. We did not find any reporting of iatrogenic injury to visceral organ, major blood vessel, or bile duct. Therefore, more operative difficulties and comparatively higher conversion rate cannot be interpreted as a lack of procedural safety.

Average hospital stay was 2.5 days. In terms of morbidity and mortality, we had a low complication rate. Postoperatively, one patient had a minor bile leak which was managed by endoscopic retrograde cholangiopancreatography (ERCP) and common bile duct (CBD) stenting. Intraoperatively or postoperatively none of the patients developed any cardiac or respiratory complication and no mortality occurred. Although in some comparative studies of elderly vs young patients a higher morbidity has been reported in elderly patients, ${ }^{17}$ a low complication rate has been reported by Marcari et al. even in octogenarians. ${ }^{18}$ Therefore, age is not a contraindication for the procedure. Patient selection, considering risk and comorbid conditions besides timely conversion of the procedure, and weighing the intraoperative difficulties affect the outcomes of procedure. This study being retrospective in nature, exclusion and inclusion criteria, i.e., proper selection criteria, could not be determined exactly, and this was the limitation of the study. Further studies are required to determine patient selection criteria and hence to improve the outcome of the procedure.

\section{Conclusion}

Procedure of laparoscopic cholecystectomy is safe in elderly patients. Operative challenges can be managed by adequate experience and timely conversion. Proper patient selection by preoperative assessment can minimize the risk of complications.

\section{References}

1. Coelho JC, Bonilha R, Pitaki SA, et al. Prevalence of gallstones in a Brazilian population. Int Surg 1999;84(1):25-28.

2. Festi D, Dormi A, Capodicasa S, et al. Incidence of gallstone disease in Italy: results from a multicenter, population-based Italian study. World J Gastroenterol 2008;14(34):5282-5289. DOI: 10.3748/wjg.14.5282.

3. Völzke H, Baumeister SE, Alte D. Independent risk factors for gallstone formation in a region with high cholelithiasis prevalence. Digestion 2005;71(2):97-105. DOI: 10.1159/000084525.

4. Bergamaschi R, Arnaud J. Immediately recognizable benefits and drawbacks after laparoscopic colon resection for benign disease. Surg Endosc 1997;11(8):802-804. DOI: 10.1007/s004649900457. 
5. Pessaux P, Tuech JJ, Derouet N, et al. Laparoscopic cholecystectomy in the elderly: a prospective study. Surg Endosc 2000;14(11):1067-1069. DOI: $10.1007 / \mathrm{s} 004640000267$.

6. Shaffer EA. Epidemiology and risk factors for gallstone disease: has the paradigm changed in the 21st century? Curr Gastroenterol Rep 2005;7(2):132-140. DOI: 10.1007/s11894-005-0051-8.

7. Bates AT, Divino C. Laparoscopic surgery in the elderly: a review of the literature. Aging Dis 2015;6(2):149-155. DOI: 10.14336/AD.2014. 0429.

8. Safran DB, Orlando R. Physiologic effects of pneumoperitoneum. Am J Surg 1994;167(2):281-286. DOI: 10.1016/0002-9610(94) 90094-9.

9. O'Leary E, Hubbard K, Tormey W, et al. Laparoscopic cholecystectomy: haemodynamic and neuroendocrine responses after pneumoperitoneum and changes in position. Br J Anaesth 1996;76(5):640-644. DOI: 10.1093/bja/76.5.640.

10. Struthers AD, Cuschieri A. Cardiovascular consequences of laparoscopic surgery. Lancet 1998;352(9127):568-570. DOI: 10.1016/ S0140-6736(97)11478-7.

11. Polychronidis A, Botaitis S, Tsaroucha A. Laparoscopic cholecystectomy in elderly patients. J Gastrointestin Liver Dis 2008;17(3):309-313.
12. Simopoulos C, Botaitis S, Polychronidis A, et al. Risk factors for conversion of laparoscopic cholecystectomy to open cholecystectomy. Surg Endosc 2005;19(7):905-909. DOI: 10.1007/s00464-004-2197-0.

13. Kaushik R. Bleeding complications in laparoscopic cholecystectomy: incidence, mechanisms, prevention and management. J Minim Access Surg 2010;6(3):59-65. DOI: 10.4103/0972-9941.68579.

14. Palanivelu C. Laparoscopic cholecystectomy. In: Parthasarthi R. Art of laparoscopic Surgery, 1st ed., vol. 1 India: Jaya Publications; 2005; p. 570.

15. Yetkin G, Uludag M, Oba S, et al. Laparoscopic cholecystectomy in elderly patients. JSLS 2009;13(4):587-591. DOI: 10.4293/108680809X 1258998404604.

16. Agrusa A, Romano G, Frazzetta G, et al. Role and outcomes of laparoscopic cholecystectomy in the elderly. Int J Surg 2014;12(Suppl 2):S37-S39. DOI: 10.1016/j.ijsu.2014.08.385.

17. Qasaimeh GR, Banihani MN. Laparoscopic cholecystectomy in the elderly and young: a comparative study. Hepatogastroenterology 2012;59(113):22-25. DOI: 10.5754/hge10198.

18. Marcari RS, Lupinacci RM, Nadal LR, et al. Outcomes of laparoscopic cholecystectomy in octogenarians. JSLS 2012;16(2):271-275. DOI: $10.4293 / 108680812$ X13427982376428. 